\title{
Mitotic Inheritance of PRC2-Mediated Silencing: Mechanistic Insights and Developmental Perspectives
}

OPEN ACCESS

Edited by:

Marc Libault,

University of Nebraska-Lincoln,

United States

Reviewed by:

Martin Howard,

John Innes Centre, United Kingdom

Lin Xu,

Institute of Plant Physiology

and Ecology, Shanghai Institutes for Biological Sciences (CAS), China

${ }^{*}$ Correspondence:

François Roudier

francois.roudier@ens-lyon.fr

Specialty section:

This article was submitted to

Plant Cell Biology,

a section of the journal

Frontiers in Plant Science

Received: 08 December 2019

Accepted: 19 February 2020

Published: 09 March 2020

Citation:

Hugues A, Jacobs CS and

Roudier F (2020) Mitotic Inheritance of PRC2-Mediated Silencing:

Mechanistic Insights

and Developmental Perspectives.

Front. Plant Sci. 11:262.

doi: $10.3389 /$ fpls.2020.00262

\begin{abstract}
Alice Hugues ${ }^{1,2}$, Chean Sern Jacobs ${ }^{1}$ and François Roudier ${ }^{1 *}$
${ }^{1}$ Laboratoire Reproduction et Développement des Plantes, ENS de Lyon, UCB Lyon 1, CNRS, INRAE, INRIA, Université de Lyon, Lyon, France, ${ }^{2}$ Master de Biologie, École Normale Supérieure de Lyon, Université Claude Bernard Lyon I, Université de Lyon, Lyon, France
\end{abstract}

Maintenance of gene repression by Polycomb Repressive Complex 2 (PRC2) that catalyzes the trimethylation of histone $\mathrm{H3}$ at lysine 27 (H3K27me3) is integral to the orchestration of developmental programs in most multicellular eukaryotes. Faithful inheritance of H3K27me3 patterns across replication ensures the stability of PRC2mediated transcriptional silencing over cell generations, thereby safeguarding cellular identities. In this review, we discuss the molecular and mechanistic principles that underlie H3K27me3 restoration after the passage of the replication fork, considering recent advances in different model systems. In particular, we aim at emphasizing parallels and differences between plants and other organisms, focusing on the recycling of parental histones and the replenishment of H3K27me3 patterns post-replication thanks to the remarkable properties of the PRC2 complex. We then discuss the necessity for fine-tuning this genuine epigenetic memory system so as to allow for cell fate and developmental transitions. We highlight recent insights showing that genome-wide destabilization of the H3K27me3 landscape during chromatin replication participates in achieving this flexible stability and provides a window of opportunity for subtle transcriptional reprogramming.

Keywords: polycomb repressive complex 2, H3K27me3 inheritance, epigenetic memory, chromatin, replication

\section{INTRODUCTION}

Polycomb repressive complex 2 (PRC2) is a conserved chromatin-modifying complex that catalyzes the trimethylation of lysine 27 of histone H3 (H3K27me3) (Margueron and Reinberg, 2011; Schuettengruber et al., 2017). PRC2 is composed of four core subunits that are necessary for its histone methyltransferase activity: Nurf55, suppressor of zeste 12 [Su(z)12], extra sex combs (ESC), and enhancer of zeste $[\mathrm{E}(\mathrm{z})]$, as originally identified in Drosophila. In multicellular organisms, members of these core subunits are often present in multigene families. For instance in the flowering plant Arabidopsis thaliana, the $\mathrm{Su}(\mathrm{z}) 12$ and $\mathrm{E}(\mathrm{z})$ subunits are encoded by three homologous genes, leading to several PRC2 complexes with potentially distinct biochemical properties and developmental roles (Mozgova and Hennig, 2015). Further functional diversity is brought about by an array of additional factors that direct PRC2 recruitment to specific loci or affect the activity of the complex (Yu et al., 2019). 
Polycomb repressive complex 2 activity orchestrates developmental and cellular programs by preserving the integrity of the gene expression patterns that underpin cell identity and function. Genetic and molecular evidence obtained from many organisms indicate that PRC2 activity is not required to initiate transcriptional repression but is necessary to maintain target gene repression, thereby providing a cellular memory system during development (Schuettengruber et al., 2017; Reinberg and Vales, 2018). Two remarkable properties lie at the heart of this genuine epigenetic process. First, the coupling between PRC2 writing and reading activities enables H3K27me3 self-propagation over large chromatin domains from an initially small number of nucleating nucleosomes marked by H3K27me3 (Oksuz et al., 2018; Yu et al., 2019). Second, H3K27me3 patterns are faithfully inherited from mother to daughter cells despite chromatin disassembly ahead of the replication fork that directly conflicts with the transmission of histone post-translational modifications (PTMs) to daughter cells (Annunziato, 2015; Masai and Foiani, 2018). The discoveries that parental histones are recycled and reincorporated into nascent chromatin and that $\mathrm{H} 3 \mathrm{~K} 27 \mathrm{me} 3$ levels are restored downstream of the replication fork in both animal and plant cell cultures (Xu et al., 2012; Alabert et al., 2015; Jiang and Berger, 2017) highlight the fact that the S-phase is not only about replicating DNA, but also chromatin together with its epigenetic potential (Ramachandran et al., 2017; Escobar et al., 2018; Reverón-Gómez et al., 2018; Serra-Cardona and Zhang, 2018).

The molecular mechanisms responsible for the faithful perpetuation of $\mathrm{H} 3 \mathrm{~K} 27 \mathrm{me} 3$-marked chromatin through cell division are under active investigation. Whereas strong evidence indicates that $\mathrm{H} 3 \mathrm{~K} 27 \mathrm{me} 3$ itself is the physical support of the PRC2-based memory system (Xu et al., 2012; Coleman and Struhl, 2017; Laprell et al., 2017), it might not be the only carrier of this epigenetic process in vivo (Højfeldt et al., 2018; Sharif and Koseki, 2018). The first part of this review aims at presenting current understanding of the histone recycling machinery and of the self-perpetuation properties that underlie the inheritance of $\mathrm{H} 3 \mathrm{~K} 27 \mathrm{me} 3$ in nascent chromatin. We then discuss the fact that, in addition to its remarkable stability, this memory system also needs to be flexible and that chromatin replication likely provides a window of opportunity enabling the transcriptional changes that drive cell fate decisions and developmental transitions.

\section{MOLECULAR MECHANISMS UNDERLYING THE MITOTIC INHERITANCE OF PRC2-MEDIATED REPRESSION}

\section{Recycling of H3K27me3-Marked Nucleosomes and Incorporation of Neo-Synthesized Histones Into Nascent Chromatin}

Parental nucleosomes disassembly at the replication fork (Teves and Henikoff, 2014; Annunziato, 2015; Masai and Foiani, 2018) is at odds with the perpetuation of parental $\mathrm{H} 3 \mathrm{~K} 27 \mathrm{me} 3$ patterns (Figure 1A). In order to ensure that both daughter cells inherit the same parental epigenetic information, parental H3H4 histones should be equiprobably distributed between the leading and the lagging strands downstream of the replication fork. However, the structural asymmetry of the replication fork is likely to induce a bias during the re-deposition of parental histones into nascent chromatin (Snedeker et al., 2017). Numerous studies in yeast and mammalian cells showed that cells manage to compensate for this intrinsic asymmetry via the intricate cooperation between histone chaperones and the DNA replication machinery that enables accurate recycling of parental histones together with their epigenetic marks (Hammond et al., 2017).

Recent studies uncovered strand-specific pathways of parental histone recycling in nascent chromatin (He et al., 2017; Gan et al., 2018; Petryk et al., 2018; Yu et al., 2018). The transfer of parental histones $\mathrm{H} 3-\mathrm{H} 4$ to the lagging strand relies on the synergistic action of the histone chaperone Mini-Chromosome Maintenance Protein 2 (MCM2), a subunit of the MCM helicase, chromosome transmission fidelity 4 (CTF4), and the lagging strand-specific primase DNA polymerase $\alpha$ (Pol- $\alpha$ ) (Huang et al., 2015; Gan et al., 2018; Petryk et al., 2018; Figure 1B). CTF4 anchors Pol- $\alpha$ in the vicinity of the MCM helicase, thus enabling the transfer of parental histones $\mathrm{H} 3-\mathrm{H} 4$ from MCM2 to the lagging stand via Pol- $\alpha$. The transfer of parental histones $\mathrm{H} 3-\mathrm{H} 4$ to the leading strand depends on Dpb3-4, a heterodimer associated with the leading strand-specific DNA polymerase $\epsilon$ (Pol- $\epsilon$ ) (He et al., 2017; Yu et al., 2018; Figure 1B'). Interestingly, asymmetric inheritance of parental histones H3$\mathrm{H} 4$ in fission yeast lacking either Dpb3 or Dpb4 results in loss of heterochromatin integrity and transcriptional activation, which emphasizes the functional role of epigenetic inheritance in the maintenance of genome stability and transcriptional programs (He et al., 2017; Yu et al., 2018).

Incorporation of newly synthesized $\mathrm{H} 3-\mathrm{H} 4$ dimers into nascent chromatin involves the two histone chaperones antisilencing function protein 1 (ASF-1) and chromatin assembly factor 1 (CAF-1) (Hammond et al., 2017; Figure 1C). Since ASF-1 co-binds parental H3-H4 histones together with MCM2, nucleosome assembly of neo-synthesized histones driven by ASF1 and CAF-1 might be also involved in the re-deposition of parental histones $\mathrm{H} 3-\mathrm{H} 4$ into nascent chromatin (Huang et al., 2015). Whereas the role of the Arabidopsis ASF-1 homologs AtASF1a/b remains unclear, CAF-1-dependent incorporation of newly synthesized histones is important for the efficient maintenance of histone PTM levels during replication (Jiang and Berger, 2017; Benoit et al., 2019).

Despite its efficiency, the recycling of parental $\mathrm{H} 3$ into nascent chromatin is not sufficient on its own to enable the full restoration of $\mathrm{H} 3 \mathrm{~K} 27 \mathrm{me} 3$ in daughter cells. Indeed, parental H3K27me3 level is diluted twofold after chromatin replication due to the incorporation of newly synthesized, unmethylated histones $\mathrm{H} 3-\mathrm{H} 4$ into nascent chromatin that is required to re-establish the initial density of nucleosomes (Figure 1). Therefore, faithful transmission of PRC2-mediated gene repression downstream of the 


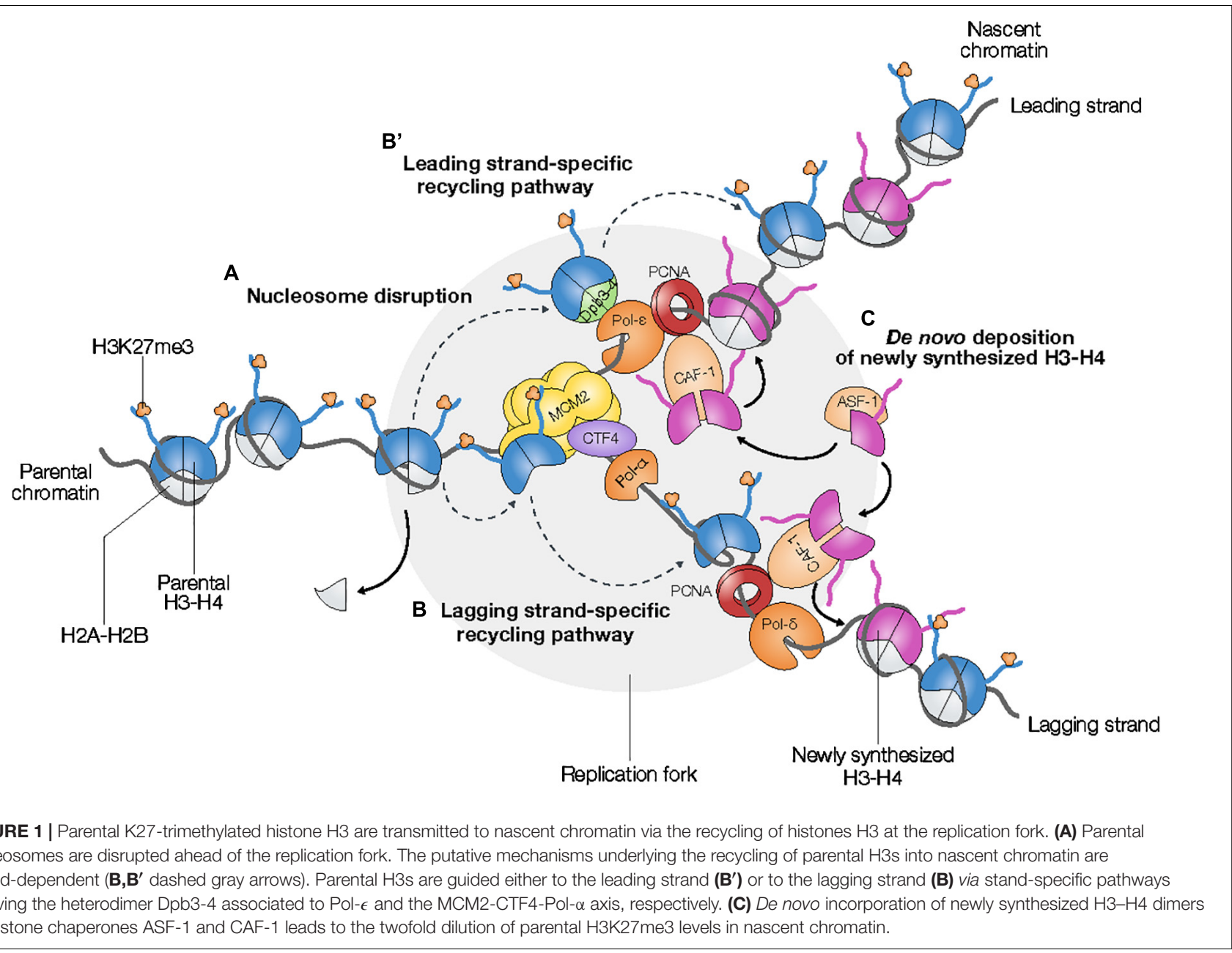

replication fork requires specific mechanisms to spread H3K27me3 from parentally modified to newly synthesized, unmodified H3 (Xu et al., 2012; Alabert et al., 2015; Jiang and Berger, 2017).

\section{Filling the Gaps? H3K27me3 Spreading Downstream of the Replication Fork What Happens to PRC2 at the Passage of the Replication Fork?}

The passage of the replication fork does not only destabilize nucleosomes but also results in the eviction of most chromatinand DNA-binding proteins, including PRC2. Nevertheless, pioneer studies based on cytological and in vitro approaches indicated that PRC2 remains localized around DNA replication sites (Hansen et al., 2008). In agreement with these observations, higher-resolution proteomic analyses showed that PRC2 is already associated to nascent chromatin immediately after the passage of the replication fork, suggesting that PRC2 is actively re-established over nascent chromatin thereby ensuring the spreading of $\mathrm{H} 3 \mathrm{~K} 27 \mathrm{me} 3$ from parental to newly synthesized, unmethylated H3 (Alabert et al., 2014; Figure 2A).
The 3D organization of chromatin might facilitate local retention of PRC2 at the replication fork

H3K27me3-marked chromatin domains have been shown to form foci in the nucleus of mammalian cells (Oksuz et al., 2018). Similarly, in plant interphasic nuclei, PRC2 is enriched in nuclear speckles containing PWWP-DOMAIN INTERACTOR OF POLYCOMBS1 (PWO1), a factor that interacts with lamin-like proteins (Mikulski et al., 2019). Although their presence in replicating cells remains to be formally established, such PRC2-enriched microenvironments could participate in maintaining PRC2 close to its targets during the passage of the fork. At a larger scale, loss of nuclear compartmentalization in the Arabidopsis 3h1 mutant lacking histone $\mathrm{H} 1$ correlates with a global diminution in H3K27me3 occupancy. This indicates that the 3D organization of chromatin into subnuclear domains probably contributes to the maintenance of H3K27me3 (Rutowicz et al., 2019), although its direct impact during replication needs to be clarified.

PRC2 binds several components of the replication machinery Interaction of PRC2 with components of the replication machinery likely contributes to its retention at the replication 
A

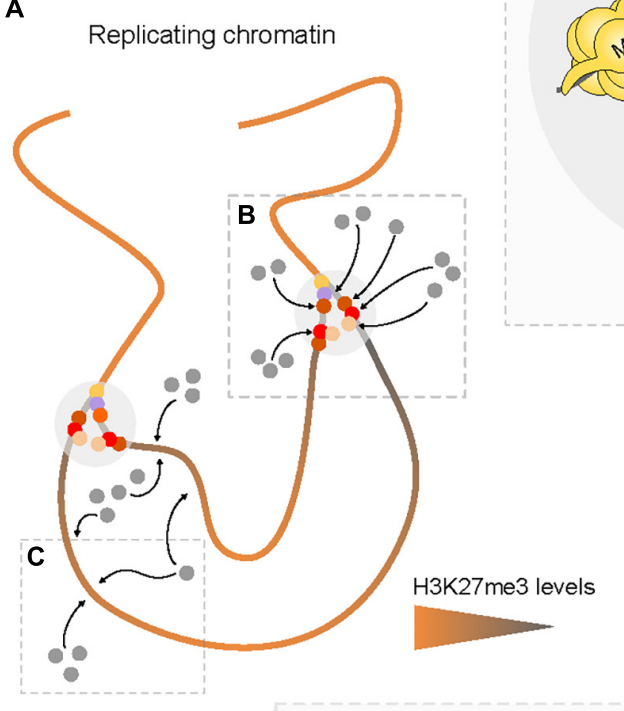

C

\section{:}

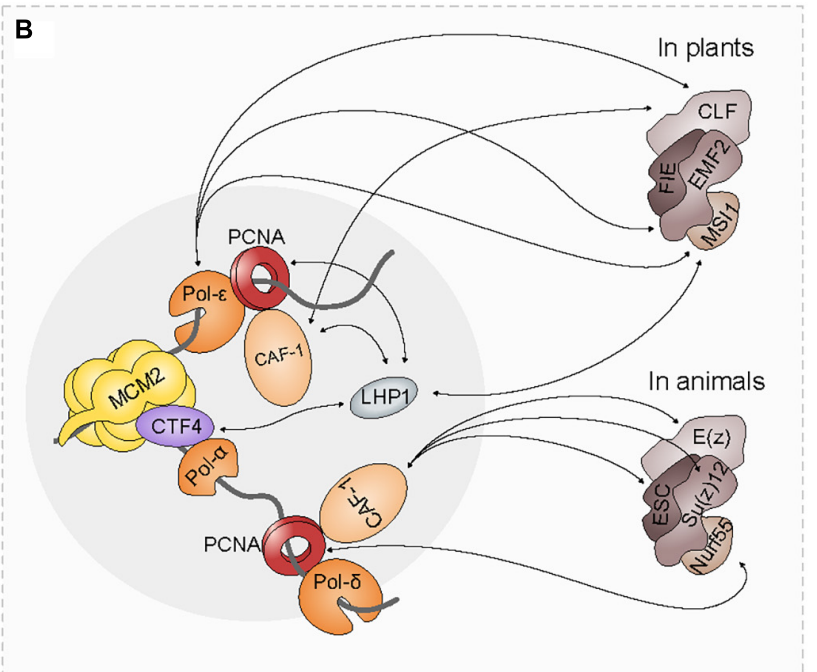

Self-propagation model

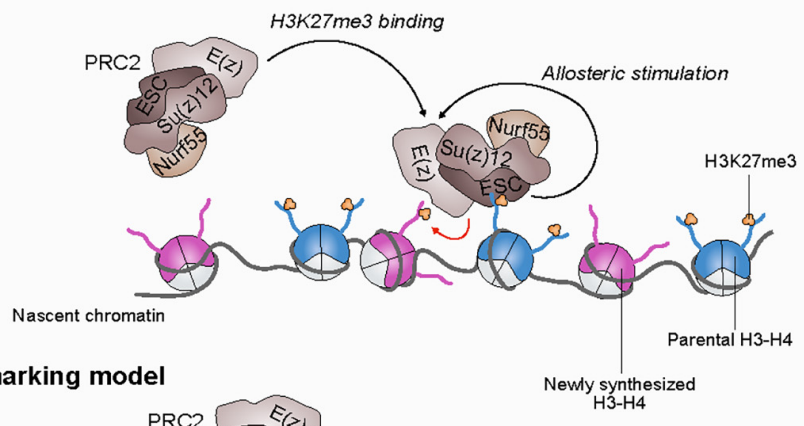

Bookmarking model

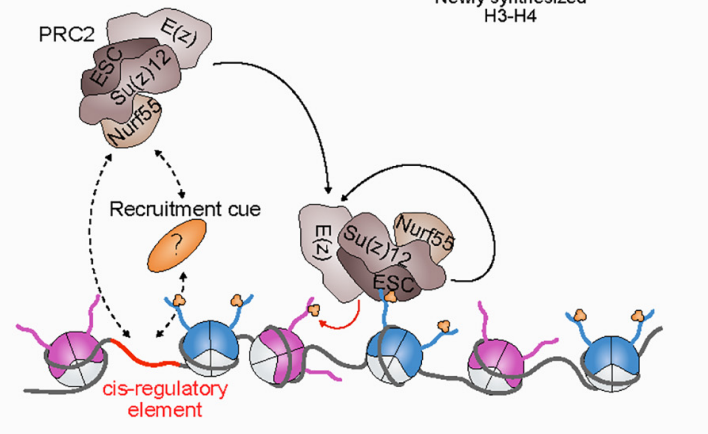

FIGURE 2 | PRC2 recruitment at the replication fork and in nascent chromatin enables faithful restoration of the H3K27me3 landscape. (A) In replicating chromatin, H3K27me3 levels (represented by a colored gradient) are diluted twofold during replication. PRC2 (gray dots) recruitment at the fork via components of the replication machinery (colored dots) (B) and in nascent chromatin (C) facilitates the restoration of parental H3K27me3 levels. (C), Top) According to the self-propagation model, the positive feedback loop between PRC2 and H3K27me3 is sufficient to propagate H3K27me3 from inherited to newly synthesized H3, thereby ensuring the maintenance of PRC2-mediated transcriptional silencing through cell division. (C, Bottom) Cis-regulatory elements might also play a critical role for the restoration of $\mathrm{H} 3 \mathrm{~K} 27 \mathrm{me} 3$ patterns by enhancing directly or indirectly the recruitment of PRC2 to nascent chromatin. 
fork (Figure 2B). PRC2 interacts with the proliferating cell nuclear antigen (PCNA) protein through CAF-1 in both plants and animals (Jiang and Berger, 2017; Cheng et al., 2019). Loss of CAF-1 induces strong developmental abnormalities including homeotic transformations in Drosophila that are reminiscent of the phenotypic defects observed in mutants lacking PRC2 activity (Anderson et al., 2011), defects in cell identity maintenance in both mouse embryonic stem cells (ESCs) and Arabidopsis meristems (Kaya et al., 2001; Clémot et al., 2018) as well as increased reprogramming abilities in mouse cells (Cheloufi et al., 2015; Cheloufi and Hochedlinger, 2017). In addition, lack of CAF-1 in Drosophila imaginal discs is associated with a massive decrease of H3K27me3 levels (Anderson et al., 2011; Yee et al., 2019), suggesting that CAF-1 activity is required for the inheritance of H3K27me3 in vivo.

Moreover, plant-specific interactions between PRC2 subunits and distinct DNA polymerases have been recently reported in Arabidopsis. Thus, loss of interaction between PRC2 and EARLY IN SHORT DAYS (ESD7), the catalytic

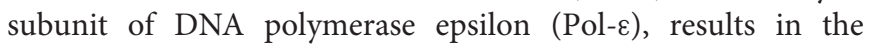
misexpression of major flowering time regulators such as AGAMOUS (AG), SUPPRESSOR OF OVEREXPRESSION OF CO 1 (SOC1), and FLOWERING LOCUS T (FT) (del Olmo et al., 2010, 2016), whose expression is controlled by PRC2. Loss of other DNA polymerases including Pol- $\alpha$ INCURVATA2 (ICU2) and Pol- $\delta$ POLD2 also impacts on H3K27me3 distribution, though a direct link with PRC2 subunits remains to be demonstrated (Pedroza-Garcia et al., 2019). These results provide evidence that interactions of PRC2 with multiple DNA polymerases acting at the replication fork likely play a significant role in the maintenance of H3K27me3 landscapes in plants.

The plant-specific protein LIKE HETEROCHROMATIN PROTEIN1 (LHP1) is required for the maintenance of H3K27me3 levels in dividing cells via its interaction with the PRC2 subunit MULTICOPY SUPPRESSOR OF IRA 1 (MSI1), a plant homolog of Nurf55 (Derkacheva et al., 2013; Veluchamy et al., 2016; Feng and Lu, 2017) and is involved in H3K27me3 spreading (Yang et al., 2017). LHP1 also binds several components of the replication fork such as CAF1 (Li and Luan, 2011; Jiang and Berger, 2017), the CTF4 homolog ENHANCER OF LHP1 (EOL1) (Zhou et al., 2017b), and possibly ICU2 (Barrero et al., 2007; Hyun et al., 2013). Hence, by providing an interaction platform for PRC2 at the replication fork, LHP1 might strengthen the coupling between parental histones recycling and $\mathrm{H} 3 \mathrm{~K} 27 \mathrm{me} 3$ spreading into nascent chromatin.

Furthermore, LHP1 interacts with components of the plant PRC1 that catalyzes H2A monoubiquitylation (Mozgova and Hennig, 2015). Given its conserved interplay with PRC2 (Yu et al., 2019), PRC1 could be involved in the maintenance of H3K27me3 patterns during replication. Indeed, in vitro studies showed that Drosophila PRC1 components remain bound to chromatin during replication (Francis et al., 2009) and that $\mathrm{H} 2 \mathrm{~A}$ monoubiquitinylation stimulates human PRC2 activity through AEBP2, a mammalian accessory PRC2 subunit (Kalb et al., 2014). In plants, absence of the PRC1 components RING1A/B causes a reduction in H3K27me3 levels over some PRC2 targets (Zhou et al., 2017a). These data indicate therefore that PRC1 could participate, via LHP1, in the recruitment of PRC2 and stimulate its activity at the replication fork.

Taken together, the aforementioned observations suggest that PRC2 is locally retained at the replication fork through multiple and dynamic interactions with components of the replication fork. These synergistic actions likely facilitate the immediate spreading of H3K27me3 over nascent chromatin, thereby participating in the inheritance of transcriptional repression, possibly in a locus-dependent manner (Figure 2B).

\section{The Self-Propagation Model: Inherited Parental H3K27me3 Instructs Its Own Spreading Onto Nascent Chromatin}

The multimeric structure of PRC2 confers two complementary activities to the complex that are essential for the selfperpetuation of $\mathrm{H} 3 \mathrm{~K} 27 \mathrm{me} 3$ patterns. Indeed, in addition to the H3K27 trimethylation (write) function, PRC2 is also able to read $\mathrm{H} 3 \mathrm{~K} 27 \mathrm{me} 3$ via the specific binding of the ESC subunit (Hansen et al., 2008; Margueron et al., 2009). Moreover, $\mathrm{Su}(\mathrm{z}) 12$ that is not directly required for H3K27me3 binding, significantly enhances PRC2 affinity for H3K27me3 (Hansen et al., 2008). These built-in writing and reading properties of PRC2 are not independent from each other since the latter enhances the catalytic activity of the former. Indeed, $\mathrm{H} 3 \mathrm{~K} 27 \mathrm{me} 3$ binding induces a conformational change of ESC that allosterically stimulates the catalytic activity of $\mathrm{E}(\mathrm{z})$ (Margueron et al., 2009). This type of positive feedback loop between chromatin readers and writers is proposed to be the hallmark property of self-sustained epigenetic memory systems (Reinberg and Vales, 2018). The ability to propagate H3K27me3 over large chromatin domains could provide robustness to PRC2mediated silencing by limiting the effect of the dilution of H3K27me3 and preventing transcriptional reactivation after replication (Xu et al., 2012). It might also compensate for the stochasticity of parental histone distribution to nascent chromatin, thereby resulting in equal spreading of the mark over daughter strands (Ramachandran and Henikoff, 2015). Thus, faithful restoration of $\mathrm{H} 3 \mathrm{~K} 27 \mathrm{me} 3$ domains after replication would largely rely on the recycling of parental H3K27me3containing nucleosomes to nascent chromatin that, in turn, instruct the recruitment of PRC2 and stimulate its writeand-read property, leading to the propagation of the mark (Figure 2C, top).

The mechanisms that spatially limit H3K27me3 spreading to preserve the boundaries of $\mathrm{H} 3 \mathrm{~K} 27 \mathrm{me} 3$ regions through DNA replication are still poorly understood (Yu et al., 2019). H3K27me3 demethylases could be involved in this process, such as the Arabidopsis Jumonji-type EARLY FLOWERING 6 (ELF6), RELATIVE OF EARLY FLOWERING 6 (REF6), and JM13 that restricts H3K27me3 domains in a tissuespecific manner (Yan et al., 2018) or the mammalian KDM6A/UTX that clears up H3K27me3 from tissuespecific enhancers (Saxena et al., 2017). Whether these 
activities directly contribute to the faithful restoration of PRC2-mediated regulation upon replication remains to be directly investigated.

\section{A Change of Paradigm? the Genomic Bookmarking Hypothesis}

The prevalence of the self-propagation model described above has been recently challenged by in vivo studies suggesting that the inheritance of parental $\mathrm{H} 3 \mathrm{~K} 27 \mathrm{me} 3$ is not always sufficient to perpetuate long-term transcriptional repression.

In Drosophila, polycomb response elements (PRE) are cis-regulatory sequences that locally recruit PRC2 and are necessary and sufficient to mediate long-term silencing (Schuettengruber et al., 2017). Whereas the function of PREs was initially associated with the nucleation of PRC2 to its targets, recent results suggest that these cis-elements might also be required for the spreading of $\mathrm{H} 3 \mathrm{~K} 27 \mathrm{me} 3$ in nascent chromatin and the maintenance of transcriptional silencing through multiple generations of cells. Indeed, excision of a PRE responsible for the repression of the nearby gene resulted in the dilution of $\mathrm{H} 3 \mathrm{~K} 27 \mathrm{me} 3$ around the excision site and in its transcriptional reactivation within few cells divisions (Coleman and Struhl, 2017; Laprell et al., 2017). However, this decrease in $\mathrm{H} 3 \mathrm{~K} 27 \mathrm{me} 3$ levels was less important than expected by passive dilution, indicating that PRC2 still maintains a roaming, PRE-independent activity (Coleman and Struhl, 2017). These results argue in favor of a model in which longterm transcriptional silencing also relies on cis-regulatory elements that act as bookmarks to anchor PRC2 at silent loci (Figure 2C, bottom). Interestingly, modeling and simulations of H3K27me3 genomic distribution in Drosophila embryos based on this bookmarking model showed that PRE excision, rather than DNA replication, can destabilize H3K27me3 domains (Michieletto et al., 2018). Understanding how many genes rely on this recruiting mechanism and at which step of the replication process such cis-regulatory elements come into play to maintain the silenced status across cell division will require further investigations.

In contrast to Drosophila, few PRE-like cis-regulatory elements have been characterized in plants (Berger et al., 2011; Förderer et al., 2016; Xiao et al., 2017; Zhou et al., 2018) and mammals (Woo et al., 2010; Schorderet et al., 2013). Furthermore, nucleosome-free and hypomethylated CpG islands in gene regulatory regions were found to act as PRC2 recruitment sites in mammals (Mendenhall et al., 2010; Lynch et al., 2012; Klose et al., 2013; Riising et al., 2014; Højfeldt et al., 2018; Oksuz et al., 2018). In vivo and in vitro deletion of such PRC2 recruitment sites within the HoxD cluster and other loci did not lead to a reduction of $\mathrm{H} 3 \mathrm{~K} 27 \mathrm{me} 3$ levels around the deletion sites (Schorderet et al., 2013; Oksuz et al., 2018), suggesting that neighboring $\mathrm{H} 3 \mathrm{~K} 27 \mathrm{me} 3$ levels may be sufficient to recruit PRC2 and maintain the mark across the cluster. However, the kinetics of $\mathrm{H} 3 \mathrm{~K} 27 \mathrm{me} 3$ deposition in mouse ESCs (mESCs) lacking a PRC2 recruitment site was slowed down compared to the wild-type situation (Oksuz et al., 2018). Similar excision experiments at endogenous loci in Arabidopsis would help determining whether such ciselements also impact on the maintenance of gene repression via PRC2 in plants.

As in Drosophila studies, recent observations in mESCs mitigate the prevalent role of $\mathrm{H} 3 \mathrm{~K} 27 \mathrm{me} 3$ in recruiting PRC2 at its genomics targets. Indeed, H3K27me3 patterns were accurately re-established via de novo methylation by PRC2 in mESCs in which H3K27me3 was totally erased from the whole genome, suggesting that additional cues are sufficient to recruit PRC2 to chromatin independently from H3K27me3 (Højfeldt et al., 2018; Figure 2C, bottom). Whether such cues are cis-encoded or result from unidentified factors associated with initial $\mathrm{H} 3 \mathrm{~K} 27 \mathrm{me} 3$ deposition remains to be determined. For instance, short non-coding RNA transcribed from $\mathrm{CpG}$ islands at genes repressed independently of PRC2 have been demonstrated to dynamically recruit $\mathrm{PRC} 2$ via $\mathrm{Su}(\mathrm{z}) 12$, thus participating to silencing maintenance (Kanhere et al., 2010).

The involvement of cis-encoded elements in conveying epigenetic memory is also suggested by observations in plant and yeast. During the vernalization process, the FLOWERING LOCUS $C$ (FLC) gene gradually acquires $\mathrm{H} 3 \mathrm{~K} 27 \mathrm{me} 3$ in a small domain of approximately three nucleosomes via de novo PRC2 recruitment. In a second phase, H3K27me3 then spreads across the whole locus in an LHP1-dependent manner to ensure long-term repression. In the $\operatorname{lhp} 1$ mutant, although spreading is affected, H3K27me3 deposition at the nucleation site is maintained across mitoses much longer than predicted if inheritance was ensured exclusively by the stochastic redistribution of parental histones (Yang et al., 2017). Epigenetic memory independent of histone inheritance has also been reported for small heterochromatin domains in yeast, the silenced state of which is inherited at a higher rate than predicted (Saxton and Rine, 2019).

Taken together, these recent advances indicate that the recruitment of $\mathrm{PRC} 2$ to nascent chromatin is likely to be even more multifactorial than initially proposed (Margueron and Reinberg, 2011), involving not only selfpropagation mechanisms but also H3K27me3-independent cues such as cis-regulatory elements and DNA-binding factors (Figure 2). As suggested by the differences observed between drosophila, mammals, and plants, these cues are likely to be both locus- and organism-dependent. These differences may reflect evolutionary changes in fine-tuning the mitotic inheritance of H3K27me3mediated transcriptional silencing in order to meet distinct developmental strategies.

\section{ROLE OF H3K27me3 INHERITANCE IN CELL FATE DECISIONS AND DEVELOPMENTAL TRANSITIONS}

Faithful maintenance of $\mathrm{H} 3 \mathrm{~K} 27 \mathrm{me} 3$ landscape provides a robust memory system that contributes to safeguard the stability of gene expression patterns, hence cell identity, 
through multiple generations. However, the coordinated and specific changes of transcriptional programs that drive cell fate acquisition entail some flexibility in this PRC2based memory. Alterations of $\mathrm{H} 3 \mathrm{~K} 27 \mathrm{me} 3$ deposition at few critical genomic loci, such as those encoding developmental regulators, can be sufficient to trigger major transcriptional changes, as recently exemplified for stomata differentiation in Arabidopsis (Lee et al., 2019). Whereas the release from PRC2 silencing can be achieved by multiple means in interphasic cells including the antagonist action of Trithorax group proteins as well as DNA-binding factors (Brand et al., 2019), recent evidence suggests that local interruption or interference with the mechanisms underlying $\mathrm{H} 3 \mathrm{~K} 27 \mathrm{me} 3$ restoration during replication could be part of the differentiation process.

\section{Time to Forget: How Chromatin Replication Might Enable Genes to Escape From PRC2-Mediated Silencing?}

Mathematical modeling suggests that transient dilution of H3K27me3 during chromatin replication weakens the stability of silent chromatin by enhancing fast-switching bistability between the silent and active states (Sneppen and Ringrose, 2019). Whereas dilution of $\mathrm{H} 3 \mathrm{~K} 27 \mathrm{me} 3$ during chromatin replication is likely to promote this instability, additional mechanisms are necessary to counteract the maintenance activities described in the previous section and potentiate the escape from PRC2 repression. Conceptually, release of PRC2 repression during replication could be achieved at a given locus through distinct, non-mutually exclusive mechanisms that would locally prevent the transfer of $\mathrm{H} 3$ parental histones into nascent chromatin, bias the distribution of parental H3K27me3 into daughter strands or hinder PRC2 recruitment or activity over nascent chromatin.

\section{Biasing H3K27me3 Inheritance to Break the Symmetry Between Daughter Chromatins?}

Recent work in Drosophila male germline stem cells showed that parental $\mathrm{H} 3$ can be locally re-deposited in a preferential manner to the leading strand during DNA replication, suggesting that asymmetric division can arise from asymmetric inheritance of parental histones (Wooten et al., 2019). Although the factors responsible for this asymmetric deposition of parental $\mathrm{H} 3$ remain to be identified, regulation of asymmetric division could rely on factors disrupting specifically one of the strand-specific recycling pathways of parental H3 (Gan et al., 2018; Petryk et al., 2018; Yu et al., 2018; Figures 1B,B' $\mathbf{B}^{\prime}$. How such a mechanism affects the inheritance of $\mathrm{H} 3 \mathrm{~K} 27 \mathrm{me} 3$-marked parental histones and whether it directly participates in asymmetric cell fate decisions requires further investigations in animals and plants.

\section{Modulation of Replication-Dependent H3K27me3 Inheritance Enables Identity Switches}

Restoration kinetics of pre-replication H3K27me3 levels may be critical to set out a window of opportunity during which a locus can escape from PRC2-mediated silencing. Interestingly, the kinetics of $\mathrm{H} 3 \mathrm{~K} 27 \mathrm{me} 3$ restoration seem to differ between plant and animal cells. In mammalian cells, H3K27me3 levels are not fully restored before the late post-mitotic $\mathrm{G} 1$ phase (Xu et al., 2012; Alabert et al., 2015). Such a slow methylation rate has been proposed to filter out fluctuations of transcription factors, thus ensuring the stability of silent states through cell divisions (Berry et al., 2017). In tobacco BY-2 cells though, H3K27me3 levels are restored as soon as early pre-mitotic G2 phase. This suggests that plant cells have evolved specific mechanisms allowing for rapid restoration of $\mathrm{H} 3 \mathrm{~K} 27 \mathrm{me} 3$ after DNA replication (Jiang and Berger, 2017). In contrast to animal cells in which PRC2 is the only complex catalyzing mono-, di-, and tri-methylation of $\mathrm{H} 3 \mathrm{~K} 27$, in plant cells a pathway involving the plant-specific H3K27 mono-methylases ATXR5/6 exist, independently from PRC2 (Jacob et al., 2014). Anchoring of both ATXR5/6 and PRC2 at the replication fork might favor a rapid restoration of $\mathrm{H} 3 \mathrm{~K} 27 \mathrm{me} 3$ after replication via the deposition of $\mathrm{H} 3 \mathrm{~K} 27 \mathrm{me} 1$ on newly synthesized nucleosome, which could serve as a template for PRC2 (Jiang and Berger, 2017). However, understanding the extent to which the kinetics of $\mathrm{H} 3 \mathrm{~K} 27 \mathrm{me} 3$ restoration impacts on the ability of cells to perpetuate PRC2-driven transcriptional memory requires further studies.

Modulation of $\mathrm{H} 3 \mathrm{~K} 27 \mathrm{me} 3$ restoration kinetics has been associated with cell fate decisions in mammalian cells. For instance, induction of mESC differentiation slows down the restoration of $\mathrm{H} 3 \mathrm{~K} 27 \mathrm{me} 3$ due to the recruitment of the H3K27me3 demethylase, ubiquitously transcribed tetratricopeptide repeat X chromosome (UTX) downstream of the replication fork, thereby preventing the spreading of H3K27me3 (Petruk et al., 2017). Slowing down the restoration of $\mathrm{H} 3 \mathrm{~K} 27 \mathrm{me} 3$ might enlarge the window of opportunity during which chromatin is in a state permissive for transcriptional reprogramming, allowing for transcription factors to bind and activate their target genes. In agreement with this, computational simulations suggest that $\mathrm{H} 3 \mathrm{~K} 27 \mathrm{me} 3$ demethylase activity during replication does favor bistability switching to the active state (Sneppen and Ringrose, 2019). Determining whether the EFL6/REF6/JMJ13 homologs also participate in such replication-coupled transcriptional reprogramming awaits direct investigations.

In addition, differentiation of ESCs is enhanced by ex14D$\mathrm{EZH} 2$, a splicing variant of EZH2 with reduced catalytic activity (Mu et al., 2018). This indicates that the presence of tightly regulated variants of PRC2 may facilitate differentiation by impeding rapid $\mathrm{H} 3 \mathrm{~K} 27 \mathrm{me} 3$ spreading in nascent chromatin at specific loci.

Finally, the equilibrium between the kinetics of H3K27 trimethylation and the mitotic rate may strongly influence the ability of a cell to perpetuate PRC2-mediated silencing through mitosis. Intuitively, if the cell cycle length is shorter than the time required for full $\mathrm{H} 3 \mathrm{~K} 27 \mathrm{me} 3$ restoration, then $\mathrm{H} 3 \mathrm{~K} 27 \mathrm{me} 3$ could be gradually lost within few cell divisions. In Arabidopsis, a coupling between the regulation of cell division timing and replication-dependent dilution of $\mathrm{H} 3 \mathrm{~K} 27 \mathrm{me} 3$ has been proposed to control the termination of the stem cell pool during floral development (Sun et al., 2014). This coupling fine-tunes the number of floral organs produced by the meristem and thus conditions the reproductive fitness of the plant. Interestingly, this mechanism might be conserved in other dicots such as 


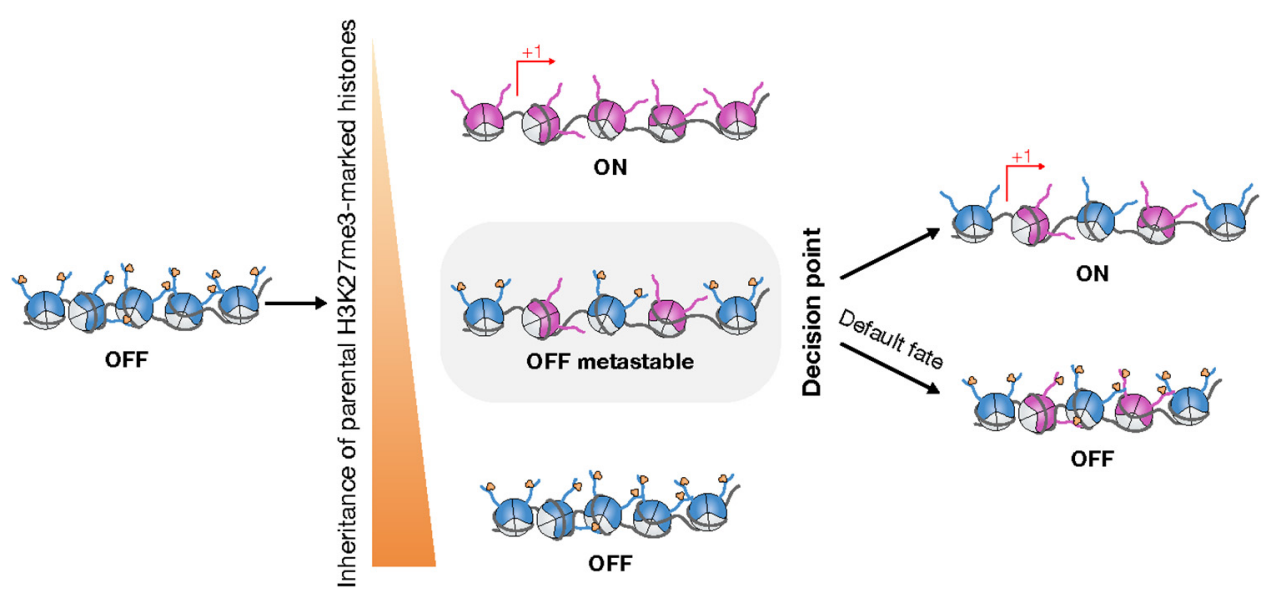

FIGURE 3 | Chromatin replication can potentiate cell fate transitions. During chromatin replication, asymmetric inheritance of parental H3K27me3 can lead to transcriptional reactivation in only one daughter cell (Middle, Top, Bottom). In case of symmetric inheritance of parental H3K27me3, repressive chromatin is switched from a stable repressive state to a metastable state that is permissive for epigenetic reprogramming (Middle). By default, H3K27me3 landscapes are restored such that repressive chromatin remains silent (Right, Bottom). Nevertheless, local disruption of the restoration of repressive chromatin can trigger specific changes in gene expression (Right, Top).

tomato (Bollier et al., 2018) and monocots such as the rice (Conrad et al., 2014).

\section{Chromatin Replication Can Potentiate PRC2-Mediated Silencing}

Whereas cells can take advantage of chromatin destabilization during the S-phase to unlock the transcription of specific PRC2 target genes, chromatin replication was also reported to potentiate PRC2-mediated silencing. In ESCs, proper establishment of $\mathrm{H} 3 \mathrm{~K} 27 \mathrm{me} 3$ at pluripotency genes such as Nanog, Oct4, and Sox2 is likely to be critical for pluripotency exit and rely on the local recruitment of PRC2 by CAF1 in S-phase (Cheng et al., 2019).

Whereas earlier studies in Arabidopsis showed that the repression of FLC established during the vernalization process is not maintained in post-mitotic cells of mature leaves (Finnegan and Dennis, 2007), replication was recently confirmed to be required for $\mathrm{H} 3 \mathrm{~K} 27 \mathrm{me} 3$ spreading and the establishment of long-term silencing of FLC (Jiang and Berger, 2017; Sharif and Koseki, 2017; Yang et al., 2017). In keeping with this, mathematical modeling revealed that the sharp switch of FLC to the silent state is consistent with a replication-dependent spreading of $\mathrm{H} 3 \mathrm{~K} 27 \mathrm{me} 3$ at the locus, suggesting that the coupling between PRC2-mediated silencing and chromatin replication can generate a quantitative integrator of environmental signals (Angel et al., 2011).

\section{CONCLUSION}

The S-phase is particularly challenging for the maintenance of transcriptional programs, hence for cell identity. Cell identity is safeguarded during chromatin replication by the inheritance of the H3K27me3 landscape, which enables the perpetuation of $\mathrm{PRC} 2-$ mediated transcriptional repression.
H3K27me3 inheritance relies on both the accurate redeposition of parental H3K27me3-marked H3 (Figure 1) and the spreading of $\mathrm{H} 3 \mathrm{~K} 27 \mathrm{me} 3$ downstream of the replication fork (Figures 2, 3). Recent advances provide new evidence that cell identity switches during developmental processes are intrinsically coupled with chromatin replication and the regulation of PRC2-mediated silencing (Figure 3). These findings strongly support the hypothesis that the S-phase opens a window of opportunity for transcriptional reprogramming and cell fate decisions.

While our understanding of the molecular mechanisms underlying $\mathrm{H} 3 \mathrm{~K} 27 \mathrm{me} 3$ inheritance is gradually increasing and points toward overall conservation between organisms, differences have also emerged in plants. Thus, separation of $\mathrm{H} 3 \mathrm{~K} 27 \mathrm{me} 1$ from $\mathrm{H} 3 \mathrm{~K} 27 \mathrm{me} 3$ catalysis into different pathways illustrates a plant-specific innovation that might directly impact on the kinetics of $\mathrm{H} 3 \mathrm{~K} 27 \mathrm{me} 3$ reestablishment during replication. In addition, developmental processes more specific to plant biology, such as continuous growth or widespread endoreplication, could introduce additional differences in the mechanistic and developmental impacts of $\mathrm{H} 3 \mathrm{~K} 27 \mathrm{me} 3$ inheritance. Further investigation of PRC2 interactions within the micro-environment of the replication fork will provide key insights to understand how H3K27me3 inheritance is modulated at specific genomic loci in order to fine-tune developmental processes in space and time.

\section{AUTHOR CONTRIBUTIONS}

$\mathrm{AH}$ and FR designed the outline of the manuscript with additional input from CJ. All authors contributed to the writing of the manuscript and approved the final manuscript. $\mathrm{AH}$ contributed to all figure designs. 


\section{ACKNOWLEDGMENTS}

Research in the Roudier Laboratory was supported by a starting grant from the Programme Avenir Lyon Saint Etienne (ANR-11-IDEX-0007) and additional core funding

\section{REFERENCES}

Alabert, C., Barth, T. K., Reverón-Gómez, N., Sidoli, S., Schmidt, A., Jensen, O. N., et al. (2015). Two distinct modes for propagation of histone PTMs across the cell cycle. Genes Dev. 29, 585-590. doi: 10.1101/gad.256354.114

Alabert, C., Bukowski-Wills, J.-C., Lee, S.-B., Kustatscher, G., Nakamura, K., de Lima Alves, F., et al. (2014). Nascent chromatin capture proteomics determines chromatin dynamics during DNA replication and identifies unknown fork components. Nat. Cell Biol. 16, 281-293. doi: 10.1038/ncb2918

Anderson, A. E., Karandikar, U. C., Pepple, K. L., Chen, Z., Bergmann, A., and Mardon, G. (2011). The enhancer of trithorax and polycomb gene Caf1/p55 is essential for cell survival and patterning in drosophila development. Dev. Camb. Engl. 138, 1957-1966. doi: 10.1242/dev.058461

Angel, A., Song, J., Dean, C., and Howard, M. (2011). A Polycomb-based switch underlying quantitative epigenetic memory. Nature 476, 105-108. doi: 10.1038/ nature 10241

Annunziato, A. T. (2015). The fork in the road: histone partitioning during DNA replication. Genes 6, 353-371. doi: 10.3390/genes6020353

Barrero, J. M., González-Bayón, R., del Pozo, J. C., Ponce, M. R., and Micol, J. L. (2007). INCURVATA2 encodes the catalytic subunit of DNA polymerase $\alpha$ and interacts with genes involved in chromatin-mediated cellular memory in Arabidopsis thaliana. Plant Cell 19, 2822-2838. doi: 10.1105/tpc.107.05 4130

Benoit, M., Simon, L., Desset, S., Duc, C., Cotterell, S., Poulet, A., et al. (2019). Replication-coupled histone H3.1 deposition determines nucleosome composition and heterochromatin dynamics during Arabidopsis seedling development. New Phytol. 221, 385-398. doi: 10.1111/nph. 15248

Berger, N., Dubreucq, B., Roudier, F., Dubos, C., and Lepiniec, L. (2011). Transcriptional regulation of Arabidopsis leafy cotyledon2 involves RLE, a cisElement that regulates trimethylation of histone $\mathrm{H} 3$ at Lysine-27[W]. Plant Cell 23, 4065-4078. doi: 10.1105/tpc.111.087866

Berry, S., Dean, C., and Howard, M. (2017). Slow chromatin dynamics allow polycomb target genes to filter fluctuations in transcription factor activity. Cell Syst. 4, 445-457.e8. doi: 10.1016/j.cels.2017.02.013

Bollier, N., Sicard, A., Leblond, J., Latrasse, D., Gonzalez, N., Gévaudant, F., et al. (2018). At-MINI ZINC FINGER2 and Sl-INHIBITOR OF MERISTEM ACTIVITY, a conserved missing link in the regulation of floral meristem termination in Arabidopsis and Tomato. Plant Cell 30, 83-100. doi: 10.1105/ tpc. 17.00653

Brand, M., Nakka, K., Zhu, J., and Dilworth, F. J. (2019). Polycomb/Trithorax antagonism: cellular memory in stem cell fate and function. Cell Stem Cell 24, 518-533. doi: 10.1016/j.stem.2019.03.005

Cheloufi, S., Elling, U., Hopfgartner, B., Jung, Y. L., Murn, J., Ninova, M., et al. (2015). The histone chaperone CAF-1 safeguards somatic cell identity. Nature 528, 218-224. doi: 10.1038/nature15749

Cheloufi, S., and Hochedlinger, K. (2017). Emerging roles of the histone chaperone CAF-1 in cellular plasticity. Curr. Opin. Genet. Dev. 46, 83-94. doi: 10.1016/j. gde.2017.06.004

Cheng, L., Zhang, X., Wang, Y., Gan, H., Xu, X., Lv, X., et al. (2019). Chromatin assembly factor 1 (CAF-1) facilitates the establishment of facultative heterochromatin during pluripotency exit. Nucleic Acids Res. 47, 11114-11131. doi: 10.1093/nar/gkz858

Clémot, M., Molla-Herman, A., Mathieu, J., Huynh, J.-R., and Dostatni, N. (2018). The replicative histone chaperone CAF1 is essential for the maintenance of identity and genome integrity in adult stem cells. Dev. Camb. Engl. 145:dev161190. doi: 10.1242/dev.161190

Coleman, R. T., and Struhl, G. (2017). Causal role for inheritance of H3K27me3 in maintaining the OFF state of a drosophila HOX gene. Science 356:eaai8236. doi: $10.1126 /$ science.aai8236 from the Ecole Normale Supérieure de Lyon as well as the Agence Nationale de la Recherche to FR. CJ was the recipient of a doctoral grant from the French Ministère de l'Enseignement Supérieur, de la Recherche et de l'Innovation.

Conrad, L. J., Khanday, I., Johnson, C., Guiderdoni, E., An, G., Vijayraghavan, U., et al. (2014). The polycomb group gene EMF2B is essential for maintenance of floral meristem determinacy in rice. Plant J. 80, 883-894. doi: 10.1111/tpj.12688

del Olmo, I., López, J. A., Vázquez, J., Raynaud, C., Piñeiro, M., and Jarillo, J. A. (2016). Arabidopsis DNA polymerase $\epsilon$ recruits components of Polycomb repressor complex to mediate epigenetic gene silencing. Nucleic Acids Res. 44, 5597-5614. doi: 10.1093/nar/gkw156

del Olmo, I., López-González, L., Martín-Trillo, M. M., Martínez-Zapater, J. M., Piñeiro, M., and Jarillo, J. A. (2010). EARLY IN SHORT DAYS 7 (ESD7) encodes the catalytic subunit of DNA polymerase epsilon and is required for flowering repression through a mechanism involving epigenetic gene silencing. Plant J. Cell Mol. Biol. 61, 623-636. doi: 10.1111/j.1365-313X.2009.04093.x

Derkacheva, M., Steinbach, Y., Wildhaber, T., Mozgová, I., Mahrez, W., Nanni, P., et al. (2013). Arabidopsis MSI1 connects LHP1 to PRC2 complexes. EMBO J. 32, 2073-2085. doi: 10.1038/emboj.2013.145

Escobar, T., Oksuz, O., Descostes, N., Bonasio, R., and Reinberg, D. (2018). Precise re-deposition of nucleosomes on repressive chromatin domains sustain epigenetic inheritance during DNA replication. BioRxiv [Preprint], doi: 10. $1101 / 418707$

Feng, J., and Lu, J. (2017). LHP1 Could Act as an Activator and a Repressor of Transcription in Plants. Front. Plant Sci. 8:2041. doi: 10.3389/fpls.2017.02041

Finnegan, E. J., and Dennis, E. S. (2007). Vernalization-induced trimethylation of histone $\mathrm{H} 3$ lysine 27 at FLC is not maintained in mitotically quiescent cells. Curr. Biol. CB 17, 1978-1983. doi: 10.1016/j.cub.2007.10.026

Förderer, A., Zhou, Y., and Turck, F. (2016). The age of multiplexity: recruitment and interactions of Polycomb complexes in plants. Curr. Opin. Plant Biol. 29, 169-178. doi: 10.1016/j.pbi.2015.11.010

Francis, N. J., Follmer, N. E., Simon, M. D., Aghia, G., and Butler, J. D., (2009). Polycomb proteins remain bound to chromatin and DNA during DNA replication in vitro. Cell 137, 110-122. doi: 10.1016/j.cell.2009.02.017

Gan, H., Serra-Cardona, A., Hua, X., Zhou, H., Labib, K., Yu, C., et al. (2018). The Mcm2-Ctf4-Pol $\alpha$ axis facilitates parental histone $\mathrm{H} 3-\mathrm{H} 4$ transfer to lagging strands. Mol. Cell 72, 140.e-151.e. doi: 10.1016/j.molcel.2018.09.001

Hammond, C. M., Strømme, C. B., Huang, H., Patel, D. J., and Groth, A. (2017). Histone chaperone networks shaping chromatin function. Nat. Rev. Mol. Cell Biol. 18, 141-158. doi: 10.1038/nrm.2016.159

Hansen, K. H., Bracken, A. P., Pasini, D., Dietrich, N., Gehani, S. S., Monrad, A., et al. (2008). A model for transmission of the H3K27me3 epigenetic mark. Nat. Cell Biol. 10, 1291-1300. doi: 10.1038/ncb1787

He, H., Li, Y., Dong, Q., Chang, A.-Y., Gao, F., Chi, Z., et al. (2017). Coordinated regulation of heterochromatin inheritance by Dpb3-Dpb4 complex. Proc. Natl. Acad. Sci. 114, 12524-12529. doi: 10.1073/pnas.1712961114

Højfeldt, J. W., Laugesen, A., Willumsen, B. M., Damhofer, H., Hedehus, L., Tvardovskiy, A., et al. (2018). Accurate H3K27 methylation can be established de novo by SUZ12-directed PRC2. Nat. Struct. Mol. Biol. 25, 225-232. doi: 10.1038/s41594-018-0036-6

Huang, H., Strømme, C. B., Saredi, G., Hödl, M., Strandsby, A., González-Aguilera, C., et al. (2015). A unique binding mode enables MCM2 to chaperone histones H3-H4 at replication forks. Nat. Struct. Mol. Biol. 22, 618-626. doi: 10.1038/ nsmb.3055

Hyun, Y., Yun, H., Park, K., Ohr, H., Lee, O., Kim, D.-H., et al. (2013). The catalytic subunit of Arabidopsis DNA polymerase $\alpha$ ensures stable maintenance of histone modification. Dev. Camb. Engl. 140, 156-166. doi: 10.1242/dev.084624

Jacob, Y., Bergamin, E., Donoghue, M. T. A., Mongeon, V., LeBlanc, C., Voigt, P., et al. (2014). Selective methylation of histone H3 variant H3.1 regulates heterochromatin replication. Science 343, 1249-1253. doi: 10.1126/science. 1248357

Jiang, D., and Berger, F. (2017). DNA replication-coupled histone modification maintains Polycomb gene silencing in plants. Science 357, 1146-1149. doi: $10.1126 /$ science.aan4965 
Kalb, R., Latwiel, S., Baymaz, H. I., Jansen, P. W. T. C., Müller, C. W., Vermeulen, M., et al. (2014). Histone H2A monoubiquitination promotes histone H3 methylation in Polycomb repression. Nat. Struct. Mol. Biol. 21, 569-571. doi: 10.1038/nsmb.2833

Kanhere, A., Viiri, K., Araújo, C. C., Rasaiyaah, J., Bouwman, R. D., Whyte, W. A., et al. (2010). Short RNAs are transcribed from repressed polycomb target genes and interact with polycomb repressive complex-2. Mol. Cell 38, 675-688. doi: 10.1016/j.molcel.2010.03.019

Kaya, H., Shibahara, K. I., Taoka, K. I., Iwabuchi, M., Stillman, B., and Araki, T. (2001). FASCIATA genes for chromatin assembly factor-1 in arabidopsis maintain the cellular organization of apical meristems. Cell 104, 131-142. doi: 10.1016/s0092-8674(01)00197-0

Klose, R. J., Cooper, S., Farcas, A. M., Blackledge, N. P., and Brockdorff, N. (2013). Chromatin sampling-an emerging perspective on targeting polycomb repressor proteins. PLoS Genet. 9:e1003717. doi: 10.1371/journal.pgen.1003717

Laprell, F., Finkl, K., and Müller, J. (2017). Propagation of Polycomb-repressed chromatin requires sequence-specific recruitment to DNA. Science 356, 85-88. doi: $10.1126 /$ science.aai8266

Lee, L. R., Wengier, D. L., and Bergmann, D. C. (2019). Cell-type-specific transcriptome and histone modification dynamics during cellular reprogramming in the Arabidopsis stomatal lineage. Proc. Natl. Acad. Sci. U.S.A. 116, 21914-21924. doi: 10.1073/pnas.1911400116

Li, H., and Luan, S. (2011). The cyclophilin AtCYP71 interacts with CAF-1 and LHP1 and functions in multiple chromatin remodeling processes. Mol. Plant 4, 748-758. doi: 10.1093/mp/ssr036

Lynch, M. D., Smith, A. J. H., De Gobbi, M., Flenley, M., Hughes, J. R., Vernimmen, D., et al. (2012). An interspecies analysis reveals a key role for unmethylated CpG dinucleotides in vertebrate Polycomb complex recruitment. EMBO J. 31, 317-329. doi: 10.1038/emboj.2011.399

Margueron, R., Justin, N., Ohno, K., Sharpe, M. L., Son, J., Drury, W. J., et al. (2009). Role of the polycomb protein EED in the propagation of repressive histone marks. Nature 461, 762-767. doi: 10.1038/nature08398

Margueron, R., and Reinberg, D. (2011). The Polycomb Complex PRC2 and its Mark in Life. Nature 469, 343-349. doi: 10.1038/nature09784

Masai, H., and Foiani, M. (2018). DNA Replication: From Old Principles to New Discoveries. Berlin: Springer.

Mendenhall, E. M., Koche, R. P., Truong, T., Zhou, V. W., Issac, B., Chi, A. S., et al. (2010). GC-rich sequence elements recruit PRC2 in mammalian ES cells. PLoS Genet. 6:e1001244. doi: 10.1371/journal.pgen.1001244

Michieletto, D., Chiang, M., Colì, D., Papantonis, A., Orlandini, E., Cook, P. R., et al. (2018). Shaping epigenetic memory via genomic bookmarking. Nucleic Acids Res. 46, 83-93. doi: 10.1093/nar/gkx1200

Mikulski, P., Hohenstatt, M. L., Farrona, S., Smaczniak, C., Stahl, Y., Kalyanikrishna, et al. (2019). The chromatin-associated protein PWO1 interacts with plant nuclear lamin-like components to regulate nuclear size. Plant Cell 31, 1141-1154. doi: 10.1105/tpc.18.00663

Mozgova, I., and Hennig, L. (2015). The polycomb group protein regulatory network. Annu. Rev. Plant Biol. 66, 269-296. doi: 10.1146/annurev-arplant043014-115627

Mu, W., Starmer, J., Yee, D., and Magnuson, T. (2018). EZH2 variants differentially regulate polycomb repressive complex 2 in histone methylation and cell differentiation. Epigenet. Chromat. 11:71. doi: 10.1186/s13072-018-0242-9

Oksuz, O., Narendra, V., Lee, C.-H., Descostes, N., LeRoy, G., Raviram, R., et al. (2018). Capturing the onset of PRC2-mediated repressive domain formation. Mol. Cell 70, 1149-1162.e5. doi: 10.1016/j.molcel.2018.05.023

Pedroza-Garcia, J.-A., De Veylder, L., and Raynaud, C. (2019). Plant DNA Polymerases. Int. J. Mol. Sci. 20:4814. doi: 10.3390/ijms20194814

Petruk, S., Cai, J., Sussman, R., Sun, G., Kovermann, S. K., Mariani, S. A., et al. (2017). Delayed accumulation of H3K27me3 on nascent DNA is essential for recruitment of transcription factors at early stages of stem cell differentiation. Mol. Cell 66, 247-257.e5. doi: 10.1016/j.molcel.2017.03.006

Petryk, N., Dalby, M., Wenger, A., Stromme, C. B., Strandsby, A., Andersson, R., et al. (2018). MCM2 promotes symmetric inheritance of modified histones during DNA replication. Science 361, 1389-1392. doi: 10.1126/science.aau0294

Ramachandran, S., Ahmad, K., and Henikoff, S. (2017). Capitalizing on disaster: establishing chromatin specificity behind the replication fork. Bioessays 39:1600150. doi: 10.1002/bies.201600150
Ramachandran, S., and Henikoff, S. (2015). Replicating nucleosomes. Sci. Adv. 1:e1500587. doi: 10.1126/sciadv.1500587

Reinberg, D., and Vales, L. D. (2018). Chromatin domains rich in inheritance. Science 361, 33-34. doi: 10.1126/science.aat7871

Reverón-Gómez, N., González-Aguilera, C., Stewart-Morgan, K. R., Petryk, N., Flury, V., Graziano, S., et al. (2018). Accurate recycling of parental histones reproduces the histone modification landscape during DNA replication. Mol. Cell. 72, 239-249.e5. doi: 10.1016/j.molcel.2018.08.010

Riising, E. M., Comet, I., Leblanc, B., Wu, X., Johansen, J. V., and Helin, K. (2014) Gene silencing triggers polycomb repressive complex 2 recruitment to $\mathrm{CpG}$ islands genome wide. Mol. Cell 55, 347-360. doi: 10.1016/j.molcel.2014.06.005

Rutowicz, K., Lirski, M., Mermaz, B., Teano, G., Schubert, J., Mestiri, I., et al. (2019). Linker histones are fine-scale chromatin architects modulating developmental decisions in Arabidopsis. Genome Biol. 20:157. doi: 10.1186/ s13059-019-1767-3

Saxena, M., Roman, A. K. S., O’Neill, N. K., Sulahian, R., Jadhav, U., and Shivdasani, R. A. (2017). Transcription factor-dependent "anti-repressive" mammalian enhancers exclude H3K27me3 from extended genomic domains. Genes Dev. 31, 2391-2404. doi: 10.1101/gad.308536.117

Saxton, D. S., and Rine, J. (2019). Epigenetic memory independent of symmetric histone inheritance. eLife 8:e51421. doi: 10.7554/eLife.51421

Schorderet, P., Lonfat, N., Darbellay, F., Tschopp, P., Gitto, S., Soshnikova, N., et al. (2013). A genetic approach to the recruitment of PRC2 at the HoxD Locus. PLoS Genet. 9:e1003951. doi: 10.1371/journal.pgen.1003951

Schuettengruber, B., Bourbon, H.-M., Croce, L. D., and Cavalli, G. (2017). Genome regulation by polycomb and trithorax: 70 Years and counting. Cell 171, 34-57. doi: 10.1016/j.cell.2017.08.002

Serra-Cardona, A., and Zhang, Z. (2018). Replication-coupled nucleosome assembly in the passage of epigenetic information and cell identity. Trends Biochem. Sci. 43, 136-148. doi: 10.1016/j.tibs.2017.12.003

Sharif, J., and Koseki, H. (2017). No winter lasts forever: polycomb complexes convert epigenetic memory of cold into flowering. Dev. Cell 42, 563-564. doi: 10.1016/j.devcel.2017.09.004

Sharif, J., and Koseki, H. (2018). Rewriting the past: de novo activity of PRC2 restores global H3K27 methylation patterns. Nat. Struct. Mol. Biol. 25:197. doi: 10.1038/s41594-018-0039-33

Snedeker, J., Wooten, M., and Chen, X. (2017). The inherent asymmetry of DNA replication. Annu. Rev. Cell Dev. Biol. 33, 291-318. doi: 10.1146/annurevcellbio-100616-060447

Sneppen, K., and Ringrose, L. (2019). Theoretical analysis of Polycomb-Trithorax systems predicts that poised chromatin is bistable and not bivalent. Nat. Commun. 10, 1-18. doi: 10.1038/s41467-019-10130-2

Sun, B., Looi, L.-S., Guo, S., He, Z., Gan, E.-S., Huang, J., et al. (2014). Timing Mechanism Dependent on Cell Division Is Invoked by Polycomb Eviction in Plant Stem Cells. Science 343:1248559. doi: 10.1126/science.1248559

Teves, S. S., and Henikoff, S. (2014). DNA torsion as a feedback mediator of transcription and chromatin dynamics. Nucleus 5, 211-218. doi: 10.4161/nucl. 29086

Veluchamy, A., Jégu, T., Ariel, F., Latrasse, D., Mariappan, K. G., Kim, S.-K., et al. (2016). LHP1 regulates $\mathrm{H} 3 \mathrm{~K} 27 \mathrm{me} 3$ spreading and shapes the three-dimensional conformation of the Arabidopsis genome. PLoS One 11:e0158936. doi: 10.1371/ journal.pone.0158936

Woo, C. J., Kharchenko, P. V., Daheron, L., Park, P. J., and Kingston, R. E. (2010). A region of the human HOXD cluster that confers polycomb-group responsiveness. Cell 140, 99-110. doi: 10.1016/j.cell.2009.12.022

Wooten, M., Snedeker, J., Nizami, Z. F., Yang, X., Ranjan, R., Urban, E., et al. (2019). Asymmetric histone inheritance via strand-specific incorporation and biased replication fork movement. Nat. Struct. Mol. Biol. 26, 732-743. doi: 10.1038/s41594-019-0269-z

Xiao, J., Jin, R., and Wagner, D. (2017). Developmental transitions: integrating environmental cues with hormonal signaling in the chromatin landscape in plants. Genome Biol. 18:88. doi: 10.1186/s13059-017-1228-9

Xu, M., Wang, W., Chen, S., and Zhu, B. (2012). A model for mitotic inheritance of histone lysine methylation. EMBO Rep. 13, 60-67. doi: 10.1038/embor.2011. 206

Yan, W., Chen, D., Smaczniak, C., Engelhorn, J., Liu, H., Yang, W., et al. (2018). Dynamic and spatial restriction of Polycomb activity by 
plant histone demethylases. Nat. Plants 4:681. doi: 10.1038/s41477-0180219-5

Yang, H., Berry, S., Olsson, T. S. G., Hartley, M., Howard, M., and Dean, C. (2017). Distinct phases of Polycomb silencing to hold epigenetic memory of cold in Arabidopsis. Science 357, 1142-1145. doi: 10.1126/science.aan 1121

Yee, W. B., Delaney, P. M., Vanderzalm, P. J., Ramachandran, S., and Fehon, R. G. (2019). The CAF-1 complex couples hippo pathway target gene expression and DNA replication. Mol. Biol. Cell 30, 2929-2942. doi: 10.1091/mbc.E19-07-0387

Yu, C., Gan, H., Serra-Cardona, A., Zhang, L., Gan, S., Sharma, S., et al. (2018). A mechanism for preventing asymmetric histone segregation onto replicating DNA strands. Science 361, 1386-1389. doi: 10.1126/science.aat8849

Yu, J.-R., Lee, C.-H., Oksuz, O., Stafford, J. M., and Reinberg, D. (2019). PRC2 is high maintenance. Genes Dev. 33, 903-935. doi: 10.1101/gad.3250 50.119

Zhou, Y., Romero-Campero, F. J., Gómez-Zambrano, Á, Turck, F., and Calonje, M. (2017a). H2A monoubiquitination in Arabidopsis thaliana is generally independent of LHP1 and PRC2 activity. Genome Biol 18:69. doi: 10.1186/ s13059-017-1197-z
Zhou, Y., Tergemina, E., Cui, H., Förderer, A., Hartwig, B., Velikkakam James, G., et al. (2017b). Ctf4-related protein recruits LHP1-PRC2 to maintain H3K27me3 levels in dividing cells in Arabidopsis thaliana. Proc. Natl. Acad. Sci. U.S.A. 114, 4833-4838. doi: 10.1073/pnas.1620955114

Zhou, Y., Wang, Y., Krause, K., Yang, T., Dongus, J. A., Zhang, Y., et al. (2018). Telobox motifs recruit CLF/SWN-PRC2 for H3K27me3 deposition via TRB factors in Arabidopsis. Nat. Genet. 50, 638-644. doi: 10.1038/s41588-0180109-9

Conflict of Interest: The authors declare that the research was conducted in the absence of any commercial or financial relationships that could be construed as a potential conflict of interest.

Copyright (c) 2020 Hugues, Jacobs and Roudier. This is an open-access article distributed under the terms of the Creative Commons Attribution License (CC BY). The use, distribution or reproduction in other forums is permitted, provided the original author(s) and the copyright owner(s) are credited and that the original publication in this journal is cited, in accordance with accepted academic practice. No use, distribution or reproduction is permitted which does not comply with these terms. 